Check for updates

Cite this: Phys. Chem. Chem. Phys., 2021, 23, 19244

Received 11th May 2021, Accepted 16th August 2021

DOI: $10.1039 / d 1 c p 02077 c$

rsc.li/pccp

\title{
Determining chemical exchange rate constants in nanoemulsions using nuclear magnetic resonance $\dagger$
}

\author{
Zhaoyuan Gong, (D) $\ddagger^{a}$ Mohammad Hossein Tootoonchi, (D) $\ddagger^{b}$ \\ Christopher A. Frakers ${ }^{\mathrm{b}}$ and Jamie D. Walls (D)*a
}

\begin{abstract}
In this work, the second-order kinetics of molecules exchanging between chemically distinct microenvironments, such as those found in nanoemulsions, is studied using nuclear magnetic resonance (NMR). A unique aspect of NMR exchange studies in nanoemulsions is that the difference in molecular resonance frequencies between the two phases, which determines whether the exchange is fast, intermediate, or slow on the NMR timescale, can depend upon the emulsion droplet composition, which is also determined by the kinetic exchange constants themselves. Within the fast-exchange regime, changes in resonance frequencies and line widths with dilution were used to extract the exchange rate constants from the NMR spectra in a manner analogous to determining the kinetic parameters in NMR ligand binding experiments. As a demonstration, the kinetic exchange parameters of isoflurane release from an emulsification of isoflurane and perflurotributylamine (FC43) were determined using NMR dilution and diffusion studies.
\end{abstract}

\section{Introduction}

Emulsions are an important class of soft materials ${ }^{1}$ that are often found in foods, such as butter, milk, and salad dressings, and in industrial processes, such as in the extraction, transport, and refinement of petroleum and associated products. Emulsions typically consist of two immiscible phases where one phase is broken up into droplets that are homogeneously dispersed throughout the other phase. Characterization of an emulsion's size distribution, composition, and rheological properties are carried out using a variety of techniques, such as optical $^{2-4}$ and electron microscopies, ${ }^{5,6}$ and nuclear magnetic resonance $(\mathrm{NMR})^{7-9}$ to name just a few.

One important application of emulsions is their use as nanocarriers of pharmaceuticals. ${ }^{10,11}$ In this case, a drug, which typically comprises a sizable composition fraction of an emulsion droplet, can exchange between hydrophobic emulsion droplets

\footnotetext{
${ }^{a}$ Department of Chemistry, University of Miami, Coral Gables, FL 33146, USA. E-mail: jwalls@miami.edu

${ }^{b}$ Diabetes Research Institute, University of Miami Miller School of Medicine, Miami, FL 33124, USA

$\dagger$ Electronic supplementary information (ESI) available: Brief descriptions of sample preparation and characterization and additional information of the NMR experiments. See DOI: 10.1039/d1cp02077c

\$ These authors contributed equally to this work.

$\S$ Co-senior author.
}

and an aqueous phase. This is depicted in Fig. 1 and by the following kinetic processes:

$$
A+\text { Emul } \underset{k_{\mathrm{B}}}{\stackrel{k_{\mathrm{F}}}{\rightleftharpoons}} A_{\text {Emul }}
$$

In eqn (1), $A$ denotes a molecule, such as a drug, in the aqueous phase while $A_{\text {Emul }}$ denotes an $A$ molecule in an emulsion droplet, which is denoted by Emul. The forward process of an $A$ molecule entering an emulsion droplet is modeled by second-order kinetics with a rate constant $k_{\mathrm{F}}$, while the reverse process of an

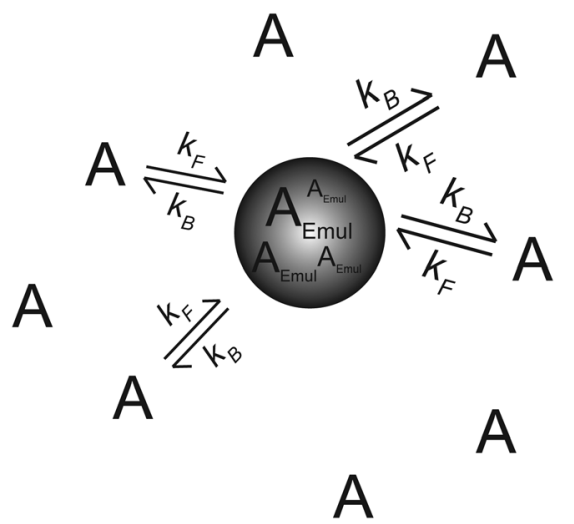

Fig. 1 An illustration of the equilibrium in eqn (1) depicting $A$ molecules in an aqueous phase exchanging with $A_{\text {Emul }}$ molecules in a spherical emulsion droplet. 
$A$ molecule leaving an emulsion droplet is modeled by firstorder kinetics with a rate constant $k_{\mathrm{B}}$. The total exchange rate constant, $k_{\text {exch }}=k_{\mathrm{F}}[$ Emul $]+k_{\mathrm{B}}$, defines a timescale of drug exchange between aqueous and organic phases given by $\tau_{\text {exch }}=\frac{1}{k_{\text {exch }}}=\frac{1}{k_{\mathrm{F}}[\text { Emul }]+k_{\mathrm{B}}}$, where [Emul] denotes the sample molar concentration of emulsion droplets.

A variety of techniques have been used to study the exchange dynamics in eqn (1), such as dialysis and related methods, ${ }^{12}$ electrochemical techniques, ${ }^{13}$ and flow cytometry. ${ }^{14}$ NMR has also been used ${ }^{15}$ to study the kinetics in eqn (1). Since most NMR studies are performed under equilibrium conditions, differences between an $A$ and $A_{\text {Emul }}$ molecule's physical properties, such as self-diffusion coefficients, ${ }^{16,17}$ and/or NMR spectral parameters, such as chemical shifts, spin-spin couplings, and transverse $\left(T_{2}\right)$ and longitudinal $\left(T_{1}\right)$ spin relaxation times, ${ }^{18,19}$ are required in order to determine $k_{\mathrm{F}}$ and $k_{\mathrm{B}}$ in eqn (1).

The Bloch-McConnell equations ${ }^{20}$ are typically used to model the effects of chemical exchange on an NMR spectrum. In general, the solutions to the Bloch-McConnell equations can be understood by simply comparing $k_{\text {exch }}$ to the effective difference in physical and/or NMR parameters between $A$ molecules in the aqueous and organic phases. Consider the situation when there is a difference in the $A$ and $A_{\text {Emul NMR }}$ resonance frequency, $\Delta \omega=\omega_{A}-\omega_{A_{\text {Emul }}} \neq 0$. In this case, $|\Delta \omega|^{-1}$ determines an effective NMR timescale in which the dynamics can be classified as being in either the $\left(\tau_{\text {exch }} \gg|\Delta \omega|^{-1}\right)$ slow-, $\left(\tau_{\text {exch }} \sim|\Delta \omega|^{-1}\right)$ intermediate-, or $\left(\tau_{\text {exch }} \ll|\Delta \omega|^{-1}\right)$ fastexchange regimes..$^{21-23}$ In all of the above exchange regimes, $|\Delta \omega|$ is usually taken to be constant. However, a small amount of $A$ molecules entering or leaving an emulsion droplet can dramatically alter the composition and chemical environment within a droplet. Since $k_{\mathrm{F}}$ and $k_{\mathrm{B}}$ in eqn (1) determine the partitioning of $A$ molecules between the emulsion droplets and the aqueous phase, both $\omega_{A_{\text {Emul }}}$ and hence $\Delta \omega$ will also depend upon $k_{\mathrm{F}}$ and $k_{\mathrm{B}}$. While traditional EXSY experiments ${ }^{24}$ could still be used to obtain the kinetic parameters in eqn (1) if the dynamics were in the slow-exchange regime, determination of $k_{\mathrm{F}}$ and $k_{\mathrm{B}}$ in the fast-exchange when $\Delta \omega$ depends upon those very rate constants would be much more challenging and has not been previously addressed.

In this work, the theory for determining the kinetic parameters of a molecule exchanging between emulsion droplets and an aqueous phase within the fast-exchange regime when $|\Delta \omega|$ depends explicitly on $k_{\mathrm{F}}$ and $k_{\mathrm{B}}$ in eqn (1) is presented. This is accomplished by first (I) calibrating the compositiondependence of an $A$ molecule's chemical shift within the emulsion droplet using a local composition model, ${ }^{25}$ and then (II) using dilution experiments to modify the forward rate of $A$ molecules entering an emulsion droplet in eqn (1). From (I) and (II), the kinetic rate constants within the fast-exchange regime can be determined from the observed chemical shifts and line widths using simple algebraic expressions that are presented in this work. As a demonstration, the theory and methodology developed in this work are applied to the problem of determining the kinetic exchange parameters of the anesthetic isoflurane in an emulsification of isoflurane in perflurotributylamine (FC43).

\section{Theory}

If the system in eqn (1) is at equilibrium, the forward and reverse rates are equal, which gives:

$$
\frac{\left[A_{\mathrm{Emul}}\right]_{\mathrm{eq}}}{[A]_{\mathrm{eq}}}=\frac{k_{\mathrm{F}}[\mathrm{Emul}]}{k_{\mathrm{B}}}=K_{\mathrm{eq}}
$$

where $[A]_{\mathrm{eq}}$ and $\left[A_{\text {Emul }}\right]_{\mathrm{eq}}$ denote the equilibrium sample molar concentrations of $A$ in the aqueous and organic phases, respectively. For a solution with a total $A$ concentration of $[A]_{\text {tot }}=[A]_{\mathrm{eq}}+\left[A_{\text {Emul }}\right]_{\mathrm{eq}}$ :

$$
\begin{aligned}
& {[A]_{\mathrm{eq}}=\frac{k_{\mathrm{B}}}{k_{\mathrm{exch}}}[A]_{\mathrm{tot}} \equiv \operatorname{Prob}_{\mathrm{aq}, \mathrm{eq}}^{A}([\text { Emul }])[A]_{\mathrm{tot}}} \\
& {\left[A_{\mathrm{Emul}}\right]_{\mathrm{eq}}=\frac{k_{\mathrm{F}}[\text { Emul }]}{k_{\mathrm{exch}}}[A]_{\mathrm{tot}} \equiv \operatorname{Prob}_{A_{\text {Emul }}, \mathrm{eq}}([\text { Emul }])[A]_{\mathrm{tot}}}
\end{aligned}
$$

where $\operatorname{Prob}_{\text {aq,eq }}^{A}([$ Emul $])$ and $\operatorname{Prob}_{A_{\text {Emul }} \text {,eq }}^{A}([$ Emul $])$ are the equilibrium probabilities that an $A$ molecule is in either the aqueous or organic phases, respectively.

Consider a simple $\frac{\pi}{2}$-acquire NMR experiment applied to a system at equilibrium and described by eqn (1). The NMR signal or free induction decay, $\operatorname{FID}(t)$, is proportional to the total transverse magnetization as a function of time after the initial $\frac{\pi}{2}$-pulse, $\operatorname{FID}(t) \propto M_{\text {tot, }+}(t)=M_{A,+}(t)+M_{A \text { Emul, }+}(t)$ where $M_{A,+}(t)=M_{A, X}(t)+i M_{A, Y}(t)$ and $M_{A \text { Emul },+}(t)=M_{A \mathrm{Emul}, X}(t)+$ $i M_{A E m u l, Y}(t)$ represent the total transverse $A$ magnetization in the aqueous and organic phases, respectively. The dynamics of both $M_{A,+}(t)$ and $M_{A E m u l,+}(t)$ can be calculated using the BlochMcConnell equations: ${ }^{20}$

$$
\frac{\mathrm{d}}{\mathrm{d} t}\left(\begin{array}{l}
M_{A,+}(t) \\
M_{A_{\mathrm{Emul}},+}(t)
\end{array}\right)=\widehat{\widehat{\mathscr{L}}}\left(\begin{array}{c}
M_{A,+}(t) \\
M_{A_{\mathrm{Emul}},+}(t)
\end{array}\right)
$$

where $\widehat{\widehat{\mathscr{L}}}$ is the evolution superoperator given by:

$$
\widehat{\widehat{\mathscr{L}}}=\left(\begin{array}{cc}
i \omega_{A}-\frac{1}{T_{2, A}^{*}}-k_{\mathrm{F}}[\text { Emul }] & k_{\mathrm{B}} \\
k_{\mathrm{F}}[\text { Emul }] & i \omega_{A_{\mathrm{Emul}}}-\frac{1}{T_{2, A_{\text {Emul }}}^{*}}-k_{\mathrm{B}}
\end{array}\right)
$$

where $\omega_{A}$ and $\omega_{A \text { Emul }}$ are the $A$ resonance frequencies (in radians per second) in the aqueous and organic phases, respectively, while $T_{2, A}^{*}$ and $T_{2, A_{\text {Emul }}^{*}}$ are the effective $A$ transverse spin relaxation times in the aqueous and organic phases, respectively.

The formal solution to eqn (4) with initial conditions $M_{A,+}(0)$ $=\operatorname{Prob}_{\mathrm{aq}, \mathrm{eq}}^{A}([$ Emul $])$ and $M_{A_{\mathrm{Emul}},+}(0)=\operatorname{Prob}_{A_{\text {Emul }} \text {,eq }}([$ Emul $])$ can be written as:

$$
\left(\begin{array}{l}
M_{A,+}(t) \\
M_{A_{\mathrm{Emul}}, t}(t)
\end{array}\right)=\mathrm{e}^{\widehat{\widehat{L}} t}\left(\begin{array}{l}
M_{A,+}(0) \\
M_{A_{\mathrm{Emul}},+}(0)
\end{array}\right)
$$


The eigenvalues of $\widehat{\widehat{\mathscr{L}}}$, which are denoted by $\lambda_{+}$ and $\lambda_{-}$, determine both the observed resonance frequencies $\left(\nu_{\mathrm{obs}}^{ \pm}=\frac{\text { Imag. }\left[\lambda_{ \pm}\right]}{2 \pi}\right)$ and line widths $\left(\Delta \nu_{\frac{1}{2}, \mathrm{obs}}^{ \pm}=-\frac{\operatorname{Real}\left[\lambda_{ \pm}\right]}{\pi}\right)$ after Fourier transformation of the $\operatorname{FID}(t)$ and are given by:

$$
\begin{aligned}
\lambda_{ \pm} & =i \frac{\omega_{\Sigma}}{2}-\frac{k_{\mathrm{exch}}}{2}-\frac{1}{2 T_{2, \Sigma}^{*}} \\
& \pm \frac{\sqrt{k_{\mathrm{exch}^{2}+\left(\frac{1}{T_{2}^{*}, \Delta}\right)^{2}+2 \frac{\Delta k}{T_{2, \Delta}^{*}}-(\Delta \omega)^{2}-2 i \Delta \omega\left(\Delta k+\frac{1}{T_{2, \Delta}^{*}}\right)}^{2}}}{}
\end{aligned}
$$

where $\omega_{\Sigma}=\omega_{A}+\omega_{A_{\text {Emul }}}, \frac{1}{T_{2, \Sigma}^{*}}=\frac{1}{T_{2, A}^{*}}+\frac{1}{T_{2, A_{\text {Emul }}}^{*}}, \frac{1}{T_{2, \Delta}^{*}}=$ $\frac{1}{T_{2, A}^{*}}-\frac{1}{T_{2, A_{\text {Emul }}}^{*}}$, and $\Delta k=k_{\mathrm{F}}[$ Emul $]-k_{\mathrm{B}}$.

\section{II.A Fast-exchange regime}

In the fast-exchange regime, the resonance at $\nu_{\text {obs }}^{-}$would have a line width of $\Delta \nu_{\frac{1}{2}, \text { obs }}^{-} \approx \frac{k_{\text {exch }}}{\pi} \gg \frac{|\Delta \omega|}{\pi}$ that is often broadened beyond the point of practical detection. Thus only the resonance at $\omega_{\text {obs }} \equiv \omega_{\text {obs }}^{+}=\operatorname{Real}\left[\lambda_{+}\right]$is typically observed in the spectrum, which is given up to order $k_{\text {exch }}^{-1}$ by:

$$
\begin{aligned}
\omega_{\mathrm{obs}} & \approx \frac{\omega_{\Sigma}}{2}-\frac{\Delta \omega \Delta k}{k_{\mathrm{exch}}}=\frac{k_{\mathrm{B}}}{k_{\mathrm{exch}}} \omega_{A}+\frac{k_{\mathrm{F}}[\text { Emul }]}{k_{\mathrm{exch}}} \omega_{A_{\text {Emul }}} \\
& =\operatorname{Prob}_{\mathrm{aq}, \mathrm{eq}}^{A}([\text { Emul }]) \omega_{A}+\operatorname{Prob}_{A_{\text {Emul }}, \mathrm{eq}}([\text { Emul }]) \omega_{A_{\mathrm{Emul}}} \equiv\langle\omega\rangle \\
& =\omega_{A}+\left(\omega_{A_{\text {Emul }}}-\omega_{A}\right) \operatorname{Prob}_{A_{\text {Emul }} \text { eq }}([\text { Emul }])
\end{aligned}
$$

In eqn (8), $\omega_{\text {obs }}$ is simply the weighted average of the $A$ resonance frequencies in the aqueous and organic phases, which is the expected result in the fast-exchange regime. ${ }^{21}$ Similarly, the observed line width, $\Delta \nu_{\frac{1}{2}}$,obs , is given up to order $k_{\text {exch }}^{-1}$ by:

$\Delta \nu_{\frac{1}{2}, \mathrm{obs}} \approx \frac{1}{\pi}\left(\left\langle\frac{1}{T_{2}^{*}}\right\rangle+\frac{(\Delta \omega)^{2} k_{\mathrm{F}} k_{\mathrm{B}}[\mathrm{Emul}]}{k_{\mathrm{exch}^{3}}{ }^{3}}\right) \equiv \frac{1}{\pi}\left(\left\langle\frac{1}{T_{2}^{*}}\right\rangle+\frac{1}{T_{2, \mathrm{exch}}}\right)$

where $\left\langle\frac{1}{T_{2}^{*}}\right\rangle=\frac{\operatorname{Prob}_{\mathrm{aq}, \mathrm{eq}}^{A}([\mathrm{Emul}])}{T_{2, A}^{*}}+\frac{\operatorname{Prob}_{A_{\mathrm{Emul}}, \mathrm{eq}}^{A}([\mathrm{Emul}])}{T_{2, A_{\text {Emul }}}^{*}}$ represents the weighted average of the effective transverse relaxation rates in the aqueous and organic phases in the absence of exchange, and

$$
\frac{1}{T_{2, \text { exch }}}=\frac{(\Delta \omega)^{2} \operatorname{Prob}_{A_{\text {Emul }}, \mathrm{eq}}^{A}([\text { Emul }])\left(1-\operatorname{Prob}_{\left.A_{\text {Emul }, \text { eq }}^{A}([\text { Emul }])\right)}\right.}{k_{\text {exch }}}
$$

is the effective transverse relaxation rate due solely to chemical exchange within the fast-exchange limit. ${ }^{21}$
In principle, both $\operatorname{Prob}_{A_{\text {Emul }} \text {,eq }}^{A}([$ Emul $])$ and $k_{\text {exch }}$ can be determined from $\Delta \nu_{\frac{1}{2}}$,obs and $\omega_{\text {obs. }}$. If $\omega_{A_{\text {Emul }}}$ is known, then from eqn (8):

$$
\operatorname{Prob}_{A_{\text {Emul }, \text { eq }}^{A}}^{A}([\text { Emul }])=\frac{\omega_{\text {obs }}-\omega_{A}}{\omega_{A_{\text {Emul }}}-\omega_{A}}
$$

However, if $\omega_{A_{\text {Emul }}}$ depends upon the composition of the emulsion droplets and hence $\operatorname{Prob}_{A_{\text {Emul }} \text { eq }}([$ Emul $])$, e.g., $\omega_{A_{\text {Emul }}}\left(\operatorname{Prob}_{A_{\text {Emul }}, \text { eq }}^{A}([\right.$ Emul $\left.])\right)$, then $\operatorname{Prob}_{A_{\text {Emul }}, \text { eq }}^{A}([$ Emul $])$ at a given [Emul] could still be determined from eqn (8) by minimizing:

$$
\begin{aligned}
& \| \omega_{\mathrm{obs}}-\omega_{A}-\operatorname{Prob}_{A_{\text {Emul }, \mathrm{eq}}}^{A}([\text { Emul }]) \\
& \quad \times\left(\omega_{A_{\text {Emul }}}\left(\operatorname{Prob}_{A_{\text {Emul }, \mathrm{eq}}}^{A}([\text { Emul }])\right) \omega_{A}\right) \| \approx 0
\end{aligned}
$$

If an $A$ molecule contains $N_{\text {nuclei }}>1$ chemically distinct NMR-active nuclei, then $\operatorname{Prob}_{A_{\text {Emul }} \text {,eq }}^{A}([$ Emul $])$ can be found by minimizing eqn (12) over all such nuclei that are observed in the NMR spectrum:

$$
\begin{aligned}
& \sum_{j=1}^{N_{\text {nuclei }}} \| \omega_{\text {obs }, j}-\omega_{A, j}-\operatorname{Prob}_{A_{\text {Emul }}, \text { eq }}^{A}([\text { Emul }]) \\
& \quad \times\left(\omega_{A_{\text {Emul }}, j}\left(\operatorname{Prob}_{A_{\text {Emul }}, \text { eq }}^{A}([\text { Emul }])\right) \omega_{A, j}\right) \| \approx 0
\end{aligned}
$$

Once $\operatorname{Prob}_{A_{\text {Emul }} \text {,eq }}^{A}([$ Emul $])$ is known, $k_{\text {exch }}$ can be determined from $\Delta \nu_{\frac{1}{2}}$,obs in eqn (9) for each observed nuclei:

$$
\begin{aligned}
k_{\mathrm{exch}} & \approx \frac{1}{N_{\text {nuclei }}} \sum_{j=1}^{N_{\text {nuclei }}} k_{\mathrm{exch}, j} \\
k_{\mathrm{exch}, j} & =\left(\omega_{A, j}-\omega_{A_{\mathrm{Emul}}, j}\left(\operatorname{Prob}_{A_{\mathrm{Emul}, \mathrm{eq}}}^{A}([\text { Emul }])\right)\right)^{2} \\
\times & \frac{\operatorname{Prob}_{A_{\mathrm{Emul}}, \mathrm{eq}}^{A}([\mathrm{Emul}])\left(1-\operatorname{Prob}_{A_{\mathrm{Emul}}, \mathrm{eq}}^{A}([\mathrm{Emul}])\right)}{\pi \Delta \nu_{\frac{1}{2}, \mathrm{obs}, j}-\frac{\operatorname{Prob}_{A_{\mathrm{Emul}}, \mathrm{eq}}^{A}([\mathrm{Emul}])}{T_{2, A_{\mathrm{Emul}}, j}^{*}}-\frac{1-\operatorname{Prob}_{A_{\mathrm{Emul}}, \mathrm{eq}}^{A}([\mathrm{Emul}])}{T_{2, A, j}^{*}}}
\end{aligned}
$$

Using $\operatorname{Prob}_{A_{\text {Emul }} \text {,eq }}^{A}([$ Emul $])$ determined from eqn (13) and $k_{\text {exch }}$ determined from eqn (14), $k_{\mathrm{F}}$ and $k_{\mathrm{B}}$ at a given [Emul] are given by:

$$
\begin{aligned}
& k_{\mathrm{F}}=k_{\mathrm{exch}} \times \frac{\operatorname{Prob}_{A_{\mathrm{Emul}}, \mathrm{eq}}^{A}([\mathrm{Emul}])}{[\mathrm{Emul}]} \\
& k_{\mathrm{B}}=k_{\mathrm{exch}} \times\left(1-\frac{\operatorname{Prob}_{A_{\mathrm{Emul}}, \mathrm{eq}}^{A}([\text { Emul }])}{[\text { Emul }]}\right)
\end{aligned}
$$

\section{Experimental}

\section{III.A Emulsion preparation and characterization}

Isoflurane in a perfluorotributylamine solution $(1: 1 \mathrm{v} / \mathrm{v})$ was emulsified in saline ( $20 \% \mathrm{v} / \mathrm{v}$ hydrophobic phase) by temperature controlled high pressure (15000 psi) homogenization using the ShearJet ${ }^{\mathrm{TM}}$ HL60 from Dyhydromatics (Maynard, MA, USA) as was 
previously reported. ${ }^{26}$ The surfactants, pluronic F68 and F127, were used in a $1: 1$ ratio to stabilize the emulsion droplets. The particle size of the emulsion droplets was characterized using dynamic light scattering (DLS). From prior studies of similar emulsions, ${ }^{27}$ dilutions greater than $40 \times$ were needed to remove particle-particle interactions and multiple scattering in order to accurately measure the hydrodynamic radius of the emulsion droplets, $r_{\text {Emul }}$. From DLS measurements at $100 \times$ dilution, $r_{\text {Emul }}=(74.7 \pm 0.7) \mathrm{nm}$. The undiluted emulsion had an initial concentration of emulsion droplets of [Emul] $\approx 172.8 \mathrm{nM}$ and an initial isoflurane concentration of $[\text { Iso }]_{\text {tot }}=(660 \pm 15) \mathrm{mM}$ that was determined from high performance liquid chromatography.

\section{III.B NMR dilution studies}

VWR Signature $^{\mathrm{TM}}$ Ergonomic High-Performance Pipettors were used to transfer a calculated amount of a stock solution of undiluted emulsion and an isoflurane saturated saline $\mathrm{D}_{2} \mathrm{O}$ solution into an NMR tube. Three replicates of each solution were prepared and measured by NMR within 12 hours of sample preparation. Experiments on a series of diluted emulsion samples were performed on a $300 \mathrm{MHz}$ Bruker AVANCE NEO spectrometer with an automatic sample changer. All proton spectra were acquired with the pulse sequence "zgcpgppr" available in the Bruker library using a $50 \mathrm{~Hz}$ presaturation pulse applied to suppress the residual water signal. The following acquisition parameters were used in the dilution experiments: a repetition time of $30 \mathrm{~s}$, a dwell time of $333 \mu \mathrm{s}$ (corresponding to a spectral width of $10 \mathrm{ppm}$ ), and an acquisition time of $3 \mathrm{~s}$. In all experiments, the transmitter frequency was set to the water resonance, and the number of scans $\left(N_{\mathrm{S}}\right)$ was varied for different dilutions to achieve a desired signal-to-noise ratio with $N_{\mathrm{S}} \leq 512$.

To obtain the isoflurane chemical shift calibration curves in an isoflurane/FC43 mixture as a function of isoflurane volume fraction, isoflurane/FC43 mixtures with an isoflurane volume fraction ranging from $10 \%$ to $100 \%$ were prepared and measured immediately in a coaxial NMR tube with the inner tube containing $\mathrm{D}_{2} \mathrm{O}$ for locking. All proton spectra for the calibration curves were acquired with the pulse sequence "zg" using a $322 \mu \mathrm{s}$ dwell time, a $3 \mathrm{~s}$ acquisition, a $50 \mathrm{~s}$ relaxation delay, and $N_{\mathrm{S}}=1$.

Finally, both $\delta_{\text {obs }}^{\alpha / \beta}$ and $\Delta \nu_{\frac{1}{2}}^{\alpha / \beta}$,obs were determined as follows: the Bloch equations were used to simulate the spectrum at each dilution by treating signals from the $\mathrm{H}^{\alpha}$ and $\mathrm{H}^{\beta}$ resonances (Fig. 2a) as a quartet and a doublet of doublets, respectively. In the simulations, the chemical shifts $\left(\delta^{\alpha / \beta}\right)$, spin-spin couplings constants $\left(J_{\mathrm{HF}}^{\alpha}\right.$ and $J_{\mathrm{HF}, 1}^{\beta}$ and $J_{\mathrm{HF}, 2}^{\beta}$ ), and the effective transverse relaxation times $\left(T_{2}^{*, \alpha / \beta}\right)$ were varied in order to minimize the absolute difference between the simulated and experimental spectra. From these simulations, both $\delta_{\text {obs }}^{\alpha / \beta} \approx \delta_{\text {optimal }}^{\alpha / \beta}$ and $\Delta \nu_{\frac{1}{2} \text {,obs }}^{\alpha / \beta} \approx \frac{1}{\pi T_{2, \text { optimal }}^{*, \alpha / \beta}}$ were determined, which were then used to determine $k_{\mathrm{F}}$ and $k_{\mathrm{B}}$ using eqn (15). It should be noted that differences in line widths for the individual peaks within a multiplet were not captured by this procedure (an example of this procedure for a single trial is given in ESI $\dagger$ ).
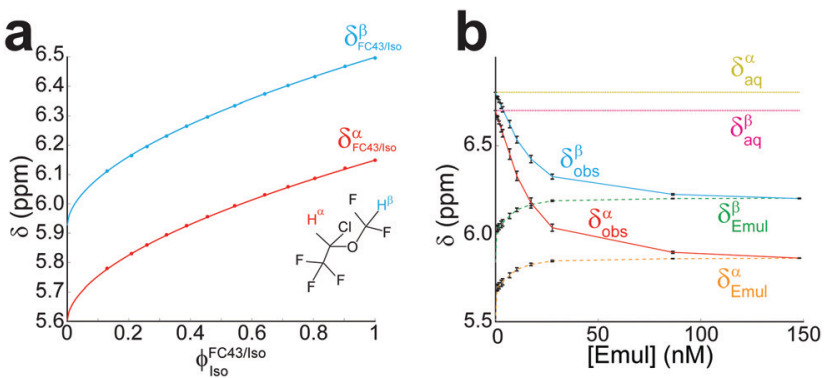

Fig. 2 (a) Chemical shifts of the (blue) $H^{\beta}$ and (red) $H^{\alpha}$ spins of isoflurane in a bulk mixture of isoflurane and $\mathrm{FC} 43$ as a function of isoflurane volume fraction, $\phi_{\mathrm{IsO}}^{\mathrm{FC} 43 / \mathrm{lso}}$. Experimental observations are denoted by asterisks while the solid curves represent the best fits to eqn (16) and (17) at $T=298 \mathrm{~K}$ with $\lambda_{\mathrm{FC} 43, \mathrm{Iso}}-\lambda_{\text {Iso, Iso }}=1220 \mathrm{~J} \mathrm{~mol}^{-1}, \delta \delta_{\mathrm{FC} 43 / \mathrm{lso}}=6.02 \mathrm{ppm}$, and $\delta_{\mathrm{FC} 43 / \mathrm{sso}}^{\alpha, \infty}=5.69 \mathrm{ppm}$. (b) In an emulsion, the observed chemical shifts ( $\delta_{\text {obs, }}^{\alpha / \beta}$, solid lines) as a function of [Emul] are shown in addition to $\delta_{\mathrm{Emul}}^{\alpha / \beta}$ calculated using the calibration curves in Fig. $2 \mathrm{a}$ and the NMR-derived $k_{\mathrm{F}}$ and $k_{\mathrm{B}}$ given in Fig. 4 . The error bars represent \pm a standard deviation after averaging over three replicates.

\section{III.C NMR diffusion studies}

All diffusion measurements were acquired on a $400 \mathrm{MHz}$ Bruker spectrometer using a stimulated echo with bipolar gradient and longitudinal eddy current delay sequence, ${ }^{28}$ which is given in Fig. 5a and was implemented using the "ledbpgp2s" pulse program in the Bruker library. Experiments were performed using 16 constant time gradients with varying amplitude $(g)$ in order to map the diffusion decay curves of the FC43 and isoflurane resonances. Different $N_{\mathrm{S}}$, gradient pulse lengths $(\delta)$, and diffusion times $(\Delta)$ were used to measure the selfdiffusion coefficients of isoflurane (using ${ }^{1} \mathrm{H}$ NMR) and FC43 in emulsion droplets (using ${ }^{19} \mathrm{~F}$ NMR) in order to achieve a desired signal to noise ratio. A $322 \mu \mathrm{s}$ dwell time, a total acquisition time of $1.5 \mathrm{~s}$, a gradient stabilization delay of $\tau=200 \mu \mathrm{s}$, an LED time of $\Delta_{\text {LED }}=5 \mathrm{~ms}$, and smoothed square shaped pulsed field gradients were used in the diffusion studies. From prior studies, ${ }^{27}$ the self-diffusion coefficient of isoflurane in pure saline solution was $D_{\text {Iso,aq }}=(9.7 \pm 0.5) \times 10^{-6} \mathrm{~cm}^{2} \mathrm{~s}^{-1}$ while in a $(1: 1 \mathrm{v} / \mathrm{v})$ isoflurane/FC43 solution, isoflurane and FC43 had self-diffusion coefficients of $D_{\text {Iso,Iso/FC43 }}=(20.34 \pm 0.06) \times$ $10^{-6} \mathrm{~cm}^{2} \mathrm{~s}^{-1}$ and $D_{\mathrm{FC} 43, \mathrm{Iso} / \mathrm{FC} 43}=(8.86 \pm 0.03) \times 10^{-6} \mathrm{~cm}^{2} \mathrm{~s}^{-1}$, respectively.

\section{Results and discussion}

In this section, the theory and methodology developed in Section II is applied to the problem of characterizing isoflurane exchange in an emulsification of isoflurane and FC43. The results are organized as follows: in Section IV.A, the composition dependence of the isoflurane chemical shifts in an isoflurane/FC43 mixture is presented, which is then used in Section IV.B to determine the isoflurane exchange parameters in an emulsion using NMR dilution experiments. In Section IV.C, NMR diffusion studies of both isoflurane and FC43 are provided, which is followed by Section IV.D with a comparison between two different 
models used in the Bloch-McConnell simulations in which the isoflurane chemical shifts in an emulsion droplet were either fixed or changed with the droplet composition. Finally in Section IV.E, a discussion of the apparent crossover between "crowded" and "lifetime-limited" regimes in NMR dilution experiments and the effects of this crossover on the NMR-determined values of $k_{\mathrm{B}}$ are discussed.

\section{IV.A Calibration of isoflurane chemical shifts in an isoflurane/FC43 solution}

As shown in Fig. 2, isoflurane consists of two ${ }^{1} \mathrm{H}$ spins, $\mathrm{H}^{\alpha}$ and $\mathrm{H}^{\beta}$, that are only coupled via scalar spin-spin interactions to neighboring ${ }^{19} \mathrm{~F}$ nuclei in the $\mathrm{CF}_{3}$ and $\mathrm{CF}_{2}$ group, respectively (the experimentally determined $J$-couplings and chemical shifts in saline and in FC43 are given in Table 1). As shown in Fig. 2a, the $\mathrm{H}^{\alpha}$ and $\mathrm{H}^{\beta}$ chemical shifts in a solution of FC43 and isoflurane, $\delta_{\mathrm{FC} 43 / \mathrm{Iso}}^{\alpha}$ and $\delta_{\mathrm{FC} 43 / \mathrm{Iso}}^{\beta}$, respectively, were found to depend upon the volume fraction of isoflurane in the mixture, $\phi_{\mathrm{Iso}}^{\mathrm{FC} 43 / \mathrm{Iso}}=\frac{V_{\mathrm{Iso}}}{V_{\mathrm{Iso}}+V_{\mathrm{FC} 43}}$. In this case, the $\phi_{\mathrm{Iso}}^{\mathrm{FC} 43 / \mathrm{Iso}}$-dependence of $\delta_{\mathrm{FC} 43 / \mathrm{Iso}}^{\alpha / \beta}$ could be fit to a local composition model ${ }^{25}$ (solid curves in Fig. 2a) given by:

$$
\delta_{\mathrm{FC} 43 / \mathrm{Iso}}^{\alpha}\left(\phi_{\mathrm{Iso}}^{\mathrm{FC} 43 / \mathrm{Iso}}\right)=\frac{\delta_{\text {Iso }}^{\alpha} \phi_{\mathrm{Iso}}^{\mathrm{FC} 43 / \mathrm{Iso}}+\Lambda \delta_{\mathrm{FC} 43 / \mathrm{Iso}}^{\alpha, \infty}\left(1-\phi_{\mathrm{Iso}}^{\mathrm{FC43/Iso}}\right)}{\phi_{\mathrm{Iso}}^{\mathrm{FC} 43 / \mathrm{Iso}}+\left(1-\phi_{\mathrm{Iso}}^{\mathrm{FC} 43 / \mathrm{Iso}}\right) \Lambda}
$$

$$
\delta_{\mathrm{FC} 43 / \mathrm{Iso}}^{\beta}\left(\phi_{\mathrm{Iso}}^{\mathrm{FC} 43 / \mathrm{Iso}}\right)=\frac{\delta_{\mathrm{Iso}}^{\beta} \phi_{\mathrm{Iso}}^{\mathrm{FC} 43 / \mathrm{Iso}}+\Lambda \delta_{\mathrm{FC} 43 / \mathrm{Iso}}^{\beta, \infty}\left(1-\phi_{\mathrm{Iso}}^{\mathrm{FC} 43 / \mathrm{Iso}}\right)}{\phi_{\mathrm{Iso}}^{\mathrm{FC} 43 / \mathrm{Iso}}+\left(1-\phi_{\mathrm{Iso}}^{\mathrm{FC} 43 / \mathrm{Iso}}\right) \Lambda}
$$

where $\Lambda=\exp \left(-\frac{\lambda_{\mathrm{FC} 43, \mathrm{Iso}}-\lambda_{\mathrm{Iso}, \mathrm{Iso}}}{R T}\right), \frac{\lambda_{\mathrm{FC} 43, \text { Iso }}-\lambda_{\text {Iso,Iso }}}{R T}$ is proportional to ratio of the difference between the FC43-isoflurane and isoflurane-isoflurane intermolecular interaction energies to thermal energy at a temperature $T, \delta_{\text {Iso }}^{\alpha}=6.146 \mathrm{ppm}$ and $\delta_{\text {Iso }}^{\beta}=6.494 \mathrm{ppm}$ are the chemical shifts in pure isoflurane, and $\delta_{\mathrm{FC} 43 / \mathrm{Iso}}^{\alpha / \beta, \infty}$ are the isoflurane chemical shifts at infinite dilution in FC43.

In Fig. $2 \mathrm{a}, \delta_{\mathrm{FC} 43 / \mathrm{Iso}}^{\alpha / \beta, \infty}$ and $\lambda_{\mathrm{FC} 43 \text {,Iso }}-\lambda_{\text {Iso,Iso }}$ were determined by minimizing the least square error between the observed chemical shifts in an FC43/isoflurane solution and those predicted by eqn (16) and (17), where the best-fit parameters

Table 1 Experimentally determined spectral parameters for isoflurane in saline and in an emulsion droplet

\begin{tabular}{lllll}
\hline Parameter & $\mathrm{H}_{\mathrm{aq}}^{\alpha}$ & $\mathrm{H}_{\mathrm{aq}}^{\beta}$ & $\mathrm{H}_{\mathrm{Emul}}^{\alpha}$ & $\mathrm{H}_{\mathrm{Emul}}^{\beta}$ \\
\hline$\delta(\mathrm{ppm})$ & $6.80^{a}$ & $6.701^{a}$ & $\mathrm{Eqn}^{a}(20)$ & $\mathrm{Eqn}(21)$ \\
$J_{\mathrm{HF}}(\mathrm{Hz})$ & $4.3^{a}$ & $71.1^{a}$ & $4.0^{b}$ & $(70.3,72.2)^{b}$ \\
$T_{2}^{*}(\mathrm{~ms})$ & $373.1^{a}$ & $385.9^{a}$ & $144.4^{b}$ & $169.9^{b}$ \\
$T_{1}(\mathrm{~s})$ & $11.20^{a}$ & $8.76^{a}$ & $2.37^{c}$ & $2.18^{b}$
\end{tabular}

${ }^{a}$ Determined from the isoflurane in saline spectrum [spectrum (12) in Fig. 3]. ${ }^{b}$ Determined from the first emulsion dilution spectrum [spectrum (1) in Fig. 3]. ${ }^{c}$ Determined from the undiluted emulsion. at $T=298 \mathrm{~K}$ were found to be $\lambda_{\mathrm{FC} 43 \text {,Iso }}-\lambda_{\text {Iso,Iso }}=1.220 \mathrm{~kJ} \mathrm{~mol}^{-1}$, $\delta_{\mathrm{FC} 43 / \mathrm{Iso}}^{\alpha, \infty}=6.02 \mathrm{ppm}$ and $\delta_{\mathrm{FC} 43 / \mathrm{Iso}}^{\beta, \infty}=5.69 \mathrm{ppm}$. The positive value for $\lambda_{\text {FC43,Iso }}-\lambda_{\text {Iso,Iso }}$ indicated that the FC43-isoflurane intermolecular interaction energies were slightly less favorable than the isoflurane-isoflurane intermolecular interaction energies.

\section{IV.B NMR measurements and determination of $k_{\mathrm{F}}$ and $\boldsymbol{k}_{\mathrm{B}}$ in an isoflurane/FC43 emulsion}

The exchange of isoflurane from an emulsification of isoflurane in FC43 was modeled analogously to the equilibrium in eqn (1):

$$
\text { Iso }+ \text { Emul } \underset{k_{\mathrm{B}}}{\stackrel{k_{\mathrm{F}}}{\rightleftharpoons}} \text { Iso }_{\text {Emul }}
$$

where "Iso" and "Iso Emul" denote isoflurane molecules in the aqueous phase and in an emulsion droplet, respectively. As noted in the previous section, a solution of isoflurane and FC43 behaves non-ideally due to differences in isoflurane-isoflurane and isoflurane-FC43 intermolecular interaction energies. The volume fraction of isoflurane in an emulsion droplet, $\phi_{\text {Iso }}^{\text {Org }}$, can be calculated from the probability of isoflurane to be in an emulsion droplet, $\operatorname{Prob}_{\text {Emul,eq }}^{\text {Iso }}([$ Emul $])=\frac{k_{\mathrm{F}}[\text { Emul }]}{k_{\text {exch }}}$ :

$$
\begin{aligned}
\phi_{\text {Iso }}^{\text {Org }} & =\frac{V_{\text {Iso }}^{\text {Org }}}{V_{\text {Iso }}^{\text {Org }}+V_{\mathrm{FC} 43}} \\
& =\frac{\operatorname{Prob}_{\text {Emul,eq }}^{\text {Iso }}([\text { Emul }]) V_{\mathrm{m}}^{\text {Iso }} V_{\text {tot }}[\text { Iso }]_{\text {tot }}}{\operatorname{Prob}_{\text {Emul }, \mathrm{eq}}^{\text {Iso }}([\text { Emul }]) V_{\mathrm{m}}^{\text {Iso }} V_{\text {tot }}[\text { Iso }]_{\text {tot }}+V_{\mathrm{FC} 43}}
\end{aligned}
$$

where [Iso] tot is the total isoflurane concentration in a volume $V_{\text {tot }}, V_{\mathrm{FC} 43}$ is the total FC43 volume, and $V_{\mathrm{m}}^{\mathrm{Iso}}=0.123329 \mathrm{~L} \mathrm{~mol}^{-1}$ is the molar volume of isoflurane at $T=298 \mathrm{~K}$.

The chemical shifts of the isoflurane resonances in an emulsion droplet were modeled by:

$$
\begin{aligned}
& \delta_{\mathrm{Emul}}^{\alpha}\left(\phi_{\mathrm{Iso}}^{\text {Org }}\right)=\delta_{\mathrm{FC} 43 / \mathrm{Iso}}^{\alpha}\left(\phi_{\text {Iso }}^{\text {Org }}\right)+\delta_{\text {offset }}^{\text {Emul }} \\
& \delta_{\mathrm{Emul}}^{\beta}\left(\phi_{\mathrm{Iso}}^{\text {Org }}\right)=\delta_{\mathrm{FC} 43 / \mathrm{Iso}}^{\beta}\left(\phi_{\text {Iso }}^{\text {Org }}\right)+\delta_{\text {offset }}^{\text {Emul }}
\end{aligned}
$$

where $\delta_{\mathrm{FC} 43 / \mathrm{Iso}}^{\alpha / \beta}\left(\phi_{\mathrm{Iso}}^{\mathrm{Org}}\right)$ are the isoflurane chemical shifts in an FC43/isoflurane mixture given in eqn (16) and (17) and eval-

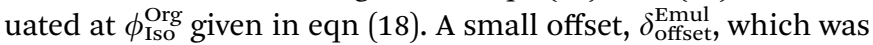
taken to be the same at all dilutions, was included in eqn (20) and (21) to account for the change in magnetic susceptibility going from a bulk isoflurane/FC43 mixture to an isoflurane/ FC43 mixture confined to small, spherical droplets stabilized by surfactants. From the dilution experiments, it was found that $\delta_{\text {offset }}^{\mathrm{Emul}}=-(0.0920 \pm 0.0002) \mathrm{ppm}$.

The chemical shifts and transverse relaxation times for isoflurane in the aqueous phase, $\delta_{\mathrm{aq}}^{\alpha}$ and $\delta_{\mathrm{aq}}^{\beta}$ and $T_{2, \mathrm{aq}}^{*, \alpha}$ and $T_{2, \mathrm{aq}}^{*, \beta}$, respectively, were determined from an isoflurane saturated saline solution while $T_{2, \mathrm{Emul}}^{*, \alpha}$ and $T_{2, \text { Emul }}^{*, \beta}$ were determined from the first emulsion dilution. These parameters are given in Table 1. As discussed earlier, the effect of exchange on an NMR spectrum depends upon the relative magnitude of $k_{\text {exch }}$ compared to the absolute frequency differences between the two 
phases, $\left|\Delta \omega^{\alpha / \beta}\left(\phi_{\text {Iso }}^{\text {Org }}\right)\right|=\gamma B_{0}\left|\delta_{\text {aq }}^{\alpha / \beta}-\delta_{\text {Emul }}^{\alpha / \beta}\left(\phi_{\text {Iso }}^{\text {Org }}\right)\right|$, where $\gamma$ is the gyromagnetic ratio, and $B_{0}$ is the magnitude of the large, static magnetic field. It is important to emphasize that a unique aspect of modeling chemical exchange for the equilibrium in eqn (18) is that $\left|\Delta \omega^{\alpha / \beta}\right|$ depends upon $\phi_{\text {Iso }}^{\text {Org }}$ and hence $k_{\text {exch }}$ [eqn (19)].

To extract $k_{\mathrm{F}}$ and $k_{\mathrm{B}}$, a series of dilution experiments of the emulsion were performed. The dilution spectra for one replicate are shown in Fig. 3, where both $\Delta \nu_{\frac{1}{2}}^{\alpha / \beta}$,obs and $\nu_{\text {obs }}^{\alpha / \beta}$ changed with dilution. Both $\nu_{\text {obs }}^{\alpha / \beta}$, which is given by the solid curves in Fig. $2 \mathrm{~b}$, and $\Delta \nu_{\frac{1}{2}}^{\alpha / \beta}$,obs , which is given in ESI, $\dagger$ were used along with $\delta_{\text {Emul }}^{\alpha / \beta}\left(\phi_{\text {Iso }}^{\text {Org }}\right)$ in eqn (20) and (21) to determine $\operatorname{Prob}_{\text {Emul,eq }}^{\text {Iso }}([$ Emul $])$ from eqn (13). Both Prob Emul,eq $_{\text {Iso }}([$ Emul $])$ and $\Delta \nu_{\frac{1}{2} \text {,obs }}^{\alpha / \beta}$ were then used to calculate $k_{\text {exch }}$ in eqn (14). From $k_{\text {exch }}$, both $k_{\mathrm{F}}$ and $k_{\mathrm{B}}$ at each [Emul] were determined using eqn (15) and are given in Fig. 4. The uncertainties in $k_{\mathrm{F}}$ and $k_{\mathrm{B}}$ in Fig. 4 mainly reflect variations between replicates since $k_{\text {exch }, \alpha}$ and $k_{\text {exch, } \beta}$ were generally within $2-13 \%$ of each other after the second dilution. While $\left|\Delta \omega^{\alpha / \beta}\right|$ increased with increasing dilution as shown in Fig. 2b, the exchange of isoflurane in the emulsion was always within the fast-exchange regime ${ }^{21-23}$ since $k_{\text {exch }} \gg\left|\Delta \omega^{\alpha / \beta}\right|$ over the entire dilution range. As a result, the isoflurane spectra in Fig. 3 contained only one set of isoflurane resonances.

Using the values of $k_{\mathrm{F}}$ and $k_{\mathrm{B}}$ in Fig. 4 , the theoretical spectra for the dilution experiments were also calculated from the

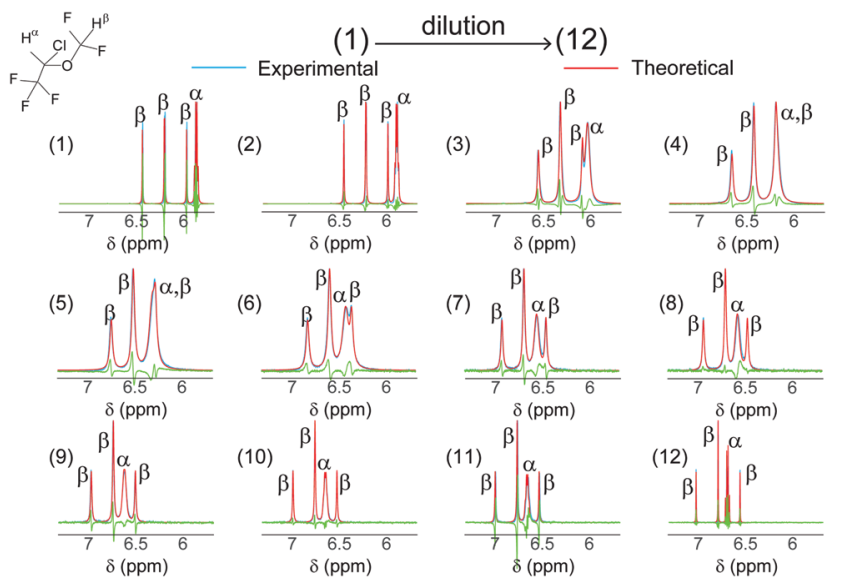

Fig. 3 (blue) Experimental ${ }^{1} \mathrm{H}$ NMR dilution spectra of isoflurane in an emulsion along with the (red) theoretical spectra calculated using the Bloch-McConnell equations ${ }^{20}$ (the green spectra represent the difference between experimental and simulated spectra). The spectra are labeled from (1) to (12) in order of increasing dilution of an initial emulsion with $[E m u l]_{0}=172.8 \mathrm{nM}$. For dilutions (1) to (12), [Emul] was given by $148 \mathrm{nM}$, $86.4 \mathrm{nM}, 27.6 \mathrm{nM}, 17.3 \mathrm{nM}, 10.4 \mathrm{nM}, 6.9 \mathrm{nM}, 3.5 \mathrm{nM}, 2.7 \mathrm{nM}, 1.7 \mathrm{nM}, 1.0 \mathrm{nM}$, $0.7 \mathrm{nM}$, and $0 \mathrm{nM}$ (corresponding to isoflurane in saline), and with corresponding scaling factors applied to the spectra given by $\times 1.0, \times 2.5$, $\times 30, \times 121, \times 277, \times 461, \times 583, \times 597, \times 469, \times 365, \times 355$, and $\times 220$, respectively. By modeling $\delta_{\mathrm{obs}}^{\alpha / \beta}$ and $\Delta \nu_{\frac{1}{2}}$,obs as a function of [Emul], both $k_{\mathrm{F}}$ and $k_{\mathrm{B}}$ in eqn (15) were determined and used as input in the BlochMcConnell simulations (red spectra).
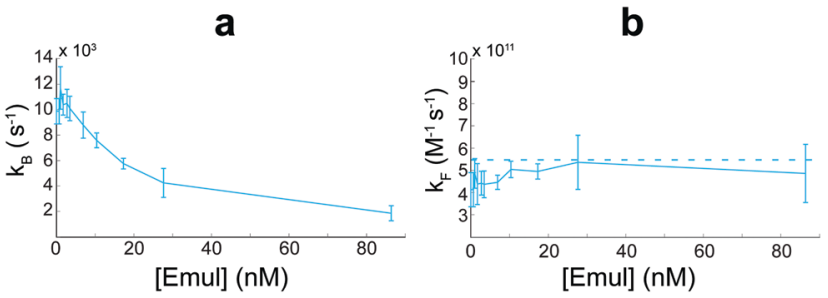

Fig. 4 The NMR determined $k_{\mathrm{F}}$ and $k_{\mathrm{B}}$ in eqn (18) as a function of [Emul] determined using $\delta_{\text {obs, }}^{\alpha / \beta} \Delta \nu_{\frac{1}{2}}^{\alpha / \beta}$,obs , and eqn (15). The error bars represent $\pm \mathrm{a}$ standard deviation after averaging the rate constants determined from both the $\mathrm{H}^{\alpha}$ and $\mathrm{H}^{\beta}$ resonances of isoflurane over three emulsion replicates. (a) As [Emul] decreased, $k_{B}$ increased by about a factor of 3 while (b) $k_{\mathrm{F}}$ was on the same order of magnitude as the corresponding Smoluchowski diffusion-limited bimolecular rate constant, ${ }^{29} k_{\text {diff }}=5.47 \times$ $10^{11} \mathrm{M}^{-1} \mathrm{~s}^{-1}$, which is represented by a dashed line.

Bloch-McConnell equations (red spectra in Fig. 3) (see ESI $\dagger$ for more details on the Bloch-McConnell simulations). The absolute differences between the simulated and observed $\delta_{\text {obs }}^{\alpha / \beta}$ and $\Delta \nu_{\frac{1}{2} \text {,obs }}^{\alpha / \beta}$ in Fig. 3 were $(0.0026 \pm 0.0020) \mathrm{ppm}$ and $(0.29 \pm 0.24) \mathrm{Hz}$, respectively, with the largest differences between the calculated and experimental spectra (the green spectra in Fig. 3) occurring at both the lowest and highest dilutions. The application of the Bloch-McConnell equations was well justified since for timescales on the order of the inverse of the frequency difference, $\frac{1}{\left|\Delta \nu_{\alpha / \beta}\right|} \approx 3.9-5.4 \mathrm{~ms}$, the root mean square displacement of isoflurane due to diffusion in the aqueous phase was $\sqrt{\frac{6 D_{\text {Iso,aq }}}{\left|\Delta \nu_{\alpha \beta}\right|}} \approx 4.8-5.6 \mu \mathrm{m}$, which was larger than the average separation between emulsion droplets, which ranged from 0.1-1.2 $\mu \mathrm{m}$ over the range of [Emul] studied in this work. The behavior of the isoflurane spectra with increasing dilution in Fig. 3 was similar to prior observations in ligand binding NMR titration experiments ${ }^{30}$ where a maximum in $\Delta \nu_{\frac{1}{2}}$ occurs at a ligand concentration such that the forward binding rate is half the ligand release rate, i.e., [ligand] $=0.5 \times \frac{k_{\mathrm{B}}}{k_{\mathrm{F}}}$. Due to the $\phi_{\text {Iso }}^{\text {Org }}$-dependence of $\delta_{\text {Emul }}^{\alpha / \beta}\left(\phi_{\text {Iso }}^{\text {Org }}\right)$, the maximal $\Delta \nu_{\frac{1}{2}}$,obs Fig. 3 occurred at a slightly larger emulsion concentration, $[$ Emul $] \approx(0.5-0.67) \times \frac{k_{\mathrm{B}}}{k_{\mathrm{F}}}$.

\section{IV.C Self-diffusion measurements}

The self-diffusion coefficients for both isoflurane and FC43 were found by fitting the experimental diffusion data (Fig. 5b, blue dots) acquired using the pulse sequence in Fig. 5a to the standard diffusion decay curve: ${ }^{31}$

$$
\frac{S(\mathbf{g})}{S(0)}=\exp \left(-4 D(\sigma \gamma \mathbf{g} \delta)^{2}\left(\Delta+\frac{(2 \kappa-2 \lambda-1) \delta}{2}-\tau\right)\right)
$$

where $\gamma$ is the gyromagnetic ratio for a given nucleus $\left({ }^{1} \mathrm{H}\right.$ or $\left.{ }^{19} \mathrm{~F}\right)$, $D$ is the self-diffusion coefficient, $\delta$ is the gradient pulse length, 


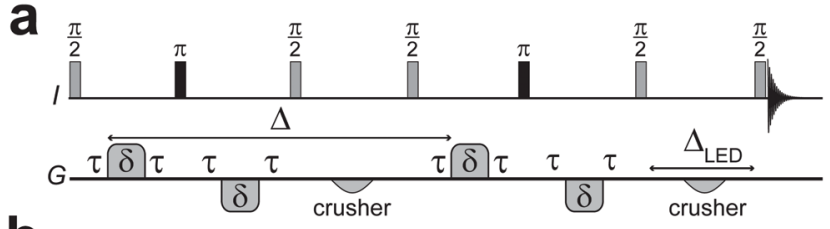

b
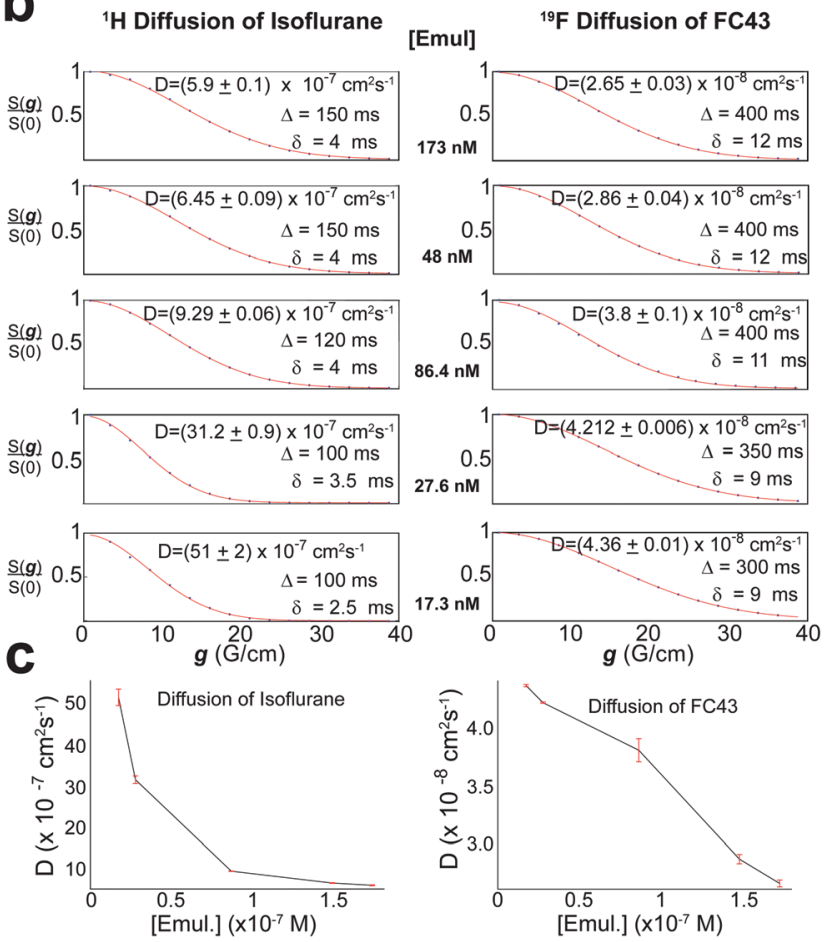

Fig. 5 Diffusion studies for a series of emulsion dilutions. (a) Stimulated echo with bipolar gradient sequence ${ }^{28}$ using smoothed rectangular gradient pulses of length $\delta$, where $\tau=200 \mu$ s is the gradient stabilization delay, $\Delta$ is the diffusion time, and $\Delta_{\text {LED }}=5 \mathrm{~ms}$ is the LED time (values of $\Delta$ and $\delta$ are given in (b)). (b) Experimental (blue asterisks) decay curves as a function of gradient strength, $g$, along with the (red curves) best fits to eqn (22) for (left) isoflurane and (right) FC43. (c) Plots of the observed self-diffusion coefficients for isoflurane and FC43 vs. [Emul], where the error bars represent \pm a standard deviation.

$\tau$ is the gradient stabilization delay, $\Delta$ is the diffusion time, and $\sigma, \kappa$, and $\lambda$ are numerical factors that are determined by the pulsed gradient pulse shape ${ }^{31}$ [for the smoothed rectangular gradient pulses used in this work $^{31}, \sigma=\frac{9}{10}, \lambda=\frac{1}{2}$, and $\left.\kappa=\frac{422}{1215}+\frac{23}{1080 \pi^{2}} \approx 0.3495\right]$. The self-diffusion coefficients of isoflurane were always over an order of magnitude larger than those of FC43 as shown in Fig. 5b. Furthermore, both isoflurane's and FC43's self-diffusion coefficients increased with increasing dilution as shown in Fig. 5c.

The isoflurane decay curves could always be fit to eqn (22) using a single self-diffusion coefficient, supporting the observation that isoflurane exchange was in the fast-exchange regime. As such, the observed isoflurane self-diffusion coefficient over the dilution range shown in Fig. $6 \mathrm{a}, D_{\text {Iso,obs, was found }}$ to be given by the average diffusion coefficient for a molecule
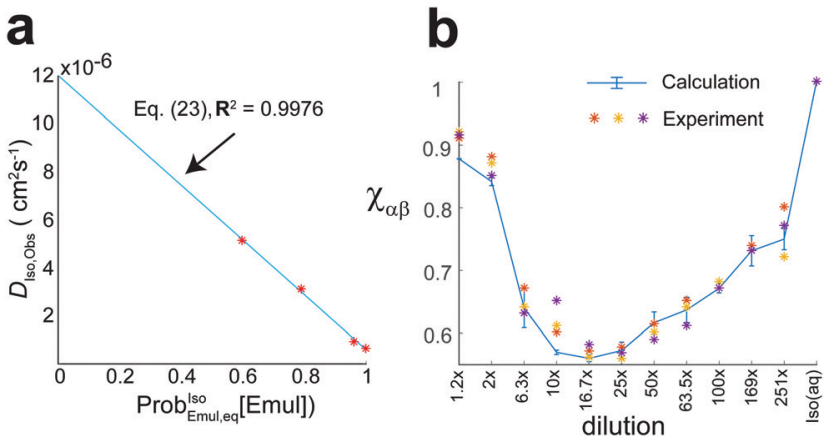

Fig. 6 (a) The observed isoflurane diffusion coefficient, $D_{\text {Iso,obs }}$ as a function of Prob ${ }_{E m m u l, e q}^{\text {Iso }}([\mathrm{Emul}])$ for data taken from Fig. 5c. A fit to eqn (23) (blue line) gave $D_{\text {Iso, saline }}^{\infty}=1.20 \times 10^{-5} \mathrm{~cm}^{2} \mathrm{~s}^{-1}$ and $D_{\text {Iso,Emul }}=5.88 \times 10^{-7} \mathrm{~cm}^{2} \mathrm{~s}^{-1}$. (b) Change in the ratio of $\mathrm{H}^{\alpha}$ and $\mathrm{H}^{\beta} \mathrm{NMR}$ signal intensity, $\chi_{\alpha \beta}$, for different dilutions and averaged over three replicates. Asterisks represent experiment data, while the solid-blue curve represents the predicted $\chi_{\alpha \beta}$ from the BlochMcConnell equations using $k_{F}$ and $k_{B}$ given in Fig. 4, with error bars representing \pm standard deviation. The observation that $\chi_{\alpha \beta}<1$ was due to both the water presaturation pulse and changing $\delta_{\text {obs }}^{\alpha / \beta}$ with dilution. In pure saline [lso(aq), rightmost], $\chi_{\alpha \beta}=1$.

exchanging between an aqueous phase and emulsion droplets in the fast-exchange regime:

$$
\begin{aligned}
D_{\text {Iso }, \mathrm{obs}}= & \langle D\rangle=\operatorname{Prob}_{\mathrm{aq}, \mathrm{eq}}^{\text {Iso }}([\text { Emul }]) D_{\text {Iso }, \text { saline }}^{\infty} \\
& +\operatorname{Prob}_{\mathrm{Emul}, \mathrm{eq}}^{\text {Iso }}([\text { Emul }]) D_{\text {Iso,Emul }} \\
= & \left(D_{\text {Iso,Emul }}-D_{\text {Iso,saline }}^{\infty}\right) \operatorname{Prob}_{\text {Emul }, \mathrm{eq}}^{\text {Iso }}([\text { Emul }]) \\
& +D_{\text {Iso,saline }}^{\infty}
\end{aligned}
$$

where Prob ${ }_{\text {Emul,eq }}^{\text {Iso }}([$ Emul $])$ was calculated using the NMR-determined $k_{\mathrm{B}}$ and $k_{\mathrm{F}}$ given in Fig. $4, D_{\text {Iso,Emul }}$ is the self-diffusion coefficient of isoflurane within an emulsion droplet, and $D_{\text {Iso,saline }}^{\infty}$ is the predicted self-diffusion coefficient of isoflurane in saline at infinite emulsion dilution, i.e., [Emul] $\rightarrow 0$. Note that from the fit to eqn (23) in Fig. $6 \mathrm{a}, D_{\text {Iso,saline }}^{\infty}=1.20 \times 10^{-5} \mathrm{~cm}^{2} \mathrm{~s}^{-1}$, which was about $24 \%$ larger than the self-diffusion coefficient of isoflurane measured in pure saline, $D_{\text {Iso,aq }}=9.7 \times 10^{-6} \mathrm{~cm}^{2} \mathrm{~s}^{-1}$.

Additionally, the ratio of NMR signal intensities for the $\mathrm{H}^{\alpha}$ and $\mathrm{H}^{\beta}$ resonances, $\chi_{\alpha \beta}=\frac{\operatorname{NMR} \text { integral }\left(H_{\alpha}\right)}{\operatorname{NMR} \text { integral }\left(\mathrm{H}_{\beta}\right)}$, was also found to change with dilution as shown in Fig. 6b. The differential attenuation of the $\mathrm{H}^{\alpha}$ and $\mathrm{H}^{\beta}$ signals, which led to $\chi_{\alpha \beta}<1$, was the result of both the presaturation pulse used to suppress the water signal and changing $\delta_{\text {obs }}^{\alpha / \beta}$ with dilution. The behavior of $\chi_{\alpha \beta}$ for different dilutions could also be calculated (solid blue curve in Fig. 6b) using the Bloch-McConnell equations with the NMR-determined $k_{\mathrm{B}}$ and $k_{\mathrm{F}}$ given in Fig. 4.

\section{IV.D Comparing exchange models with either changing or fixed $\delta_{\text {Emul }}^{\alpha / \beta}$}

While the model with $\delta_{\text {Emul }}^{\alpha / \beta}$ changing with $\phi_{\text {Iso }}^{\text {Org }}$ [eqn (20) and (21)] was shown to be consistent with the experimental results in Fig. 3 and 6 , an alternative model where $\delta_{\text {Emul }}^{\alpha / \beta}$ did not change with $\phi_{\mathrm{Iso}}^{\mathrm{Org}}$ was also found to be consistent with these experimental 
results too (see $\mathrm{ESI} \dagger$ for more details). However, the NMRdetermined $k_{\mathrm{B}}$ and $k_{\mathrm{F}}$ from both models were still within an order of magnitude from each other, $k_{\mathrm{F}} \approx 10^{11} \mathrm{M}^{-1} \mathrm{~s}^{-1}$ and $k_{\mathrm{B}} \approx 10^{4} \mathrm{~s}^{-1}$, while exhibiting a similar [Emul]-dependence. This naturally leads to the question as to whether these two models can be experimentally distinguished from one another?

One way to distinguish between the two models was to utilize the [Iso] tot $^{-d e p e n d e n c e ~ o f ~} \phi_{\text {Iso }}^{\text {Org }}$ in eqn (19). For the model used in Fig. 3 and 6, both $\delta_{\text {Emul }}^{\alpha}\left(\phi_{\text {Iso }}^{\text {Org }}\right)$ [eqn (20)] and $\delta_{\text {Emul }}^{\beta}\left(\phi_{\text {Iso }}^{\text {Org }}\right)$ [eqn (21)] explicitly depended upon $\phi_{\text {Iso }}^{\text {Org }}$ and hence on [Iso] $]_{\text {tot }}$ If [Iso] tot increased, both $\delta_{\text {Emul }}^{\alpha}$ and $\delta_{\text {Emul }}^{\beta}$ in this model would experience a downfield shift due to increasing $\phi_{\text {Iso }}^{\text {Org }}$ (Fig. 2a). However, for the model in which $\delta_{\text {Emul }}^{\alpha / \beta}$ was fixed, no spectral shifts with changing [Iso] tot would be observed. In Fig. 7a, the experimental spectrum (blue) of the inner resonances of the $\mathrm{H}^{\beta}$ multiplet in an initial sample containing $500 \mu \mathrm{L}$ of a $10 \times$ emulsion dilution is shown (solid) in addition to the spectra in which nominally (dotted) $1 \mu \mathrm{L}$ and (dashed) $2 \mu \mathrm{L}$ of pure isoflurane was added directly to the NMR tube, resulting in a slight increase in [Iso] tot from $0.0967 \mathrm{M}$ up to $0.1289 \mathrm{M}$. As a result of increasing [Iso] tot, the experimental spectra exhibited a slight downfield shift. In Fig. 7 (middle inset), the water resonance frequency did not change with changing [Iso] tot, indicating that the observed isoflurane shifts were not global

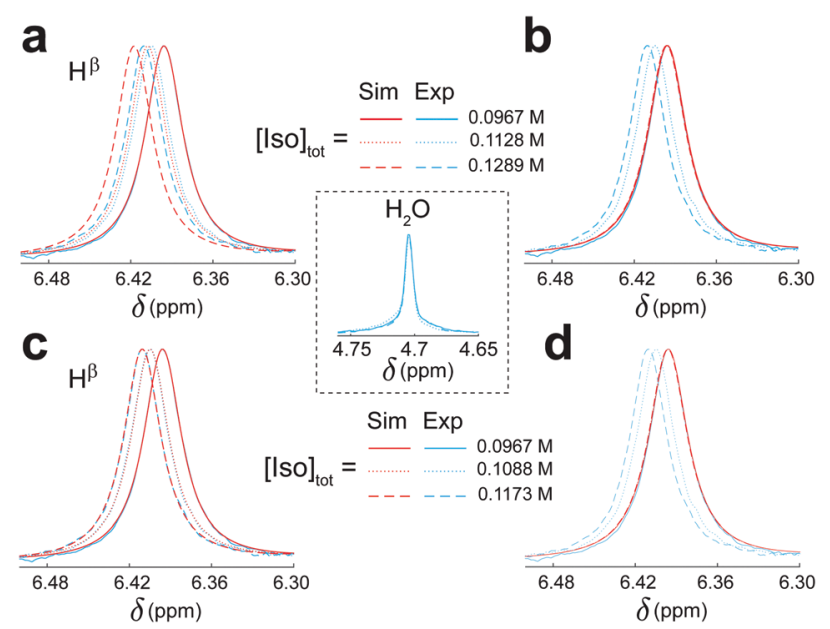

Fig. 7 Effects of [lso $]_{\text {tot }}$ on an NMR spectrum. The (blue) experimental spectrum for the inner transitions of the $\mathrm{H}^{\beta}$ multiplet are shown for different [lso] tot caused by titrating pure isoflurane into an initial $500 \mu \mathrm{L}$ sample of $10 \times$ a diluted emulsion. Experimentally, the isoflurane resonances shifted downfield with increasing [lso] tot. [middle inset] The residual $\mathrm{H}_{2} \mathrm{O}$ resonance frequency was unchanged with changing [lso] tot, indicating that the observed downfield shift was local and not global in nature. (a) Simulated spectra from the Bloch-McConnell equations using $\delta_{\text {Emul }}^{\alpha / \beta}\left(\phi_{\text {Iso }}^{\text {Org }}\right)$ in eqn (20) and (21) exhibited a similar downfield shift while (b) simulations with fixed $\delta_{\text {Emul }}^{\alpha}=5.83 \mathrm{ppm}$ and $\delta_{\text {Emul }}^{\beta}=6.19 \mathrm{ppm}$ did not. (c) Better agreement between simulation and experiment was found if it was assumed that smaller volumes of isoflurane were titrated than in a [0.76 $\mu \mathrm{L}$ and $1.28 \mu \mathrm{L}$ vs. $1 \mu \mathrm{L}$ and $2 \mu \mathrm{L}$ ]. (d) For comparison, the corresponding simulated spectra using fixed $\delta_{\text {Emul }}^{\alpha / \beta}$ also did not exhibit a downfield shift. In the Bloch-McConnell simulations, either (a and c) $k_{\mathrm{F}}=5.12 \times$ $10^{11} \mathrm{M}^{-1} \mathrm{~s}^{-1}$ and $k_{\mathrm{B}}=5.96 \times 10^{3} \mathrm{~s}^{-1}$ or $(\mathrm{b}$ and $\mathrm{d}) k_{\mathrm{F}}=4.31 \times 10^{11} \mathrm{M}^{-1} \mathrm{~s}^{-1}$ and $k_{\mathrm{B}}=3.80 \times 10^{3} \mathrm{~s}^{-1}$ were used. in nature. The results from numerical simulations of the (Fig. 7a, red spectra) using the Bloch-McConnell equations with $\delta_{\text {Emul }}^{\alpha / \beta}\left(\phi_{\text {Iso }}^{\text {Org }}\right)$ in eqn (20) and (21) showed a similar downfield shift, whereas the spectra from simulations with fixed $\delta_{\mathrm{Emul}}^{\alpha / \beta}$ remained roughly the same as shown in Fig. 7b. In the simulations, $k_{\mathrm{F}}$ and $k_{\mathrm{B}}$ were determined from $\nu_{\mathrm{obs}}^{\alpha / \beta}$ and $\Delta \nu_{\frac{1}{2}}^{\alpha / \beta}$,obs for an initial $10 \times$ dilution (solid), which gave either [Fig. 7a] $k_{\mathrm{F}}=5.12 \times$ $10^{11} \mathrm{M}^{-1} \mathrm{~s}^{-1}$ and $k_{\mathrm{B}}=5.96 \times 10^{3} \mathrm{~s}^{-1}$ or [Fig. $\left.7 \mathrm{~b}\right] k_{\mathrm{F}}=4.31 \times 10^{11}$ $\mathrm{M}^{-1} \mathrm{~s}^{-1}$ and $k_{\mathrm{B}}=3.80 \times 10^{3} \mathrm{~s}^{-1}$ for fixed $\delta_{\mathrm{Emul}}^{\alpha}=5.83 \mathrm{ppm}$ and $\delta_{\text {Emul }}^{\beta}=6.19 \mathrm{ppm}$.

The simulated spectra in Fig. 7a exhibited similar downfield shifts as the experimental spectra, albeit with larger predicted shifts than what was experimentally observed. As shown in Fig. 7c, however, better agreement between simulation and experiment could be achieved if it was assumed that the actual titrated volumes of isoflurane were slightly smaller than the nominal values of $1 \mu \mathrm{L}$ and $2 \mu \mathrm{L}$ assumed in Fig. 7a, in this case (dotted) $0.75 \mu \mathrm{L}$ and (dashed) $1.28 \mu \mathrm{L}$. The possibility that the actual amounts of isoflurane added were less than $1 \mu \mathrm{L}$ and $2 \mu \mathrm{L}$ seems physically justified due to the ineffectiveness of accurately titrating such small volumes directly into an NMR tube. For comparison, the simulated spectra for fixed $\delta_{\mathrm{Emul}}^{\alpha / \beta}$ using the same values of [Iso] tot assumed in Fig. 7c are shown in Fig. 7d, where no significant spectral changes were observed with changing [Iso] tot. Overall, the results in Fig. 7 support the use of $\delta_{\mathrm{Emul}}^{\alpha / \beta}\left(\phi_{\text {Iso }}^{\text {Org }}\right)$ in eqn (20) and (21) in modeling isoflurane exchange in these nanoemulsions.

It should be noted that both $k_{\mathrm{F}}$ and $k_{\mathrm{B}}$ in Fig. 4 were calculated using eqn (15) under the assumption that the chemical shifts, $\delta_{\text {aq }}^{\alpha / \beta}$ and $\delta_{\text {Emul }}^{\alpha / \beta}([$ Emul $])$, and the effective transverse relaxation times, $T_{2, \mathrm{aq}}^{*, \alpha / \beta}$ and $T_{2, \text { Emul }}^{*, \alpha / \beta}$, were always described over the entire dilution range by the expressions given in Table 1. Experimentally, small differences in magnetic field inhomogeneity between samples are expected to introduce small variations in $\Delta \nu_{\frac{1}{2}}^{\alpha / \beta}$,obs . Furthermore, it is possible that the chemical shifts, $T_{2}^{*} \mathrm{~s}$, and the effective ${ }^{1} \mathrm{H}-{ }^{19} \mathrm{~F}$ spin-spin interactions could also be changing with dilution in a manner that is not taken into account using the model described above. For example, while the spin-spin couplings and chemical shifts (up to a global shift of $\delta_{\text {offset }}^{\text {Emul }}$ derived from the isoflurane spectrum at high [Emul] (low dilutions) were similar to those of isoflurane in pure FC43 since Prob Emul,eq $_{\text {equl }]}([\mathrm{Emu}) \approx 1$, the spectral parameters of isoflurane in the aqueous phase could possibly differ from their isotropic values in pure saline [Table 1] due to anisotropic interactions, such as dipolar couplings ${ }^{32}$ and chemical shift anisotropy, ${ }^{33}$ that are not fully averaged away due to the partial ordering of isoflurane in concentrated emulsion samples. With dilution, these contributions would decrease to zero as the spinspin couplings and chemical shifts approached their isotropic values in the aqueous phase given in Table 1 . Furthermore, the intrinsic transverse relaxation times in both the emulsion droplets and aqueous phase could also change with dilution (say due to changes in partial ordering and/or some, yet unidentified mechanism). Further investigations employing 
additional experimental methods, such as magic-angle spinning, could be used to better characterize the contributions of anisotropic interactions in emulsions. For the system studied in this work, however, both the dilution spectra in Fig. 3 and the diffusion data in Fig. 6 a provided orthogonal measurements that were consistent with the above model using the spectral parameters in Table 1, suggesting that anisotropic interactions, if present, did not significantly affect the NMR-determined $k_{F}$ and $k_{\mathrm{B}}$ values give in Fig. 4. More details about the tolerances of the NMRdetermined $k_{\mathrm{F}}$ and $k_{\mathrm{B}}$ rate constants to errors or changes to $T_{2}^{*, \alpha / \beta}$ and $\delta^{\alpha / \beta}$ are provided in ESI. $\dagger$

\section{IV.E "Crowded" vs. "lifetime-limited" regimes}

In this section, the effects of emulsion droplet crowding on the NMR-determined $k_{\mathrm{F}}$ and $k_{\mathrm{B}}$ shown in Fig. 4 will be addressed. Earlier theoretical work ${ }^{34,35}$ predicted that bimolecular rate constants should increase with increasing volume fraction of spherical emulsion droplets, which can be calculated by:

$$
\phi=\frac{4 \pi r_{\mathrm{Emul}}{ }^{3} N_{\mathrm{A}}[\mathrm{Emul}]}{3}
$$

where $N_{\mathrm{A}}$ is Avogadro's number. While $k_{\mathrm{F}}$ increased slightly with increasing [Emul] in Fig. 4b, the increase was much smaller than predicted ${ }^{34,35}$ from prior theoretical work (see ESI $\dagger$ for more information). Furthermore, $k_{\mathrm{F}}$ was on the same order of magnitude as the Smoluchowski diffusion-limited bimolecular rate constant, ${ }^{29} k_{\text {diff }}=4 \pi r_{\text {Emul }} D_{\text {Iso,aq. }} \cdot N_{\mathrm{A}}=5.47 \times 10^{11} \mathrm{M}^{-1} \mathrm{~s}^{-1}$ (Fig. 4b, dashed line). This suggests that the rate of isoflurane entering an emulsion droplet was diffusion-limited.

As shown in Fig. 4a, on the other hand, the NMR-determined $k_{\mathrm{B}}$ changed dramatically with [Emul], increasing by roughly a factor of three for dilutions up to $50 \times$. For dilutions greater than $50 \times, k_{\mathrm{B}}$ appeared to be roughly constant at $k_{\mathrm{B}} \approx(1.05 \pm 0.07) \times$ $10^{4} \mathrm{~s}^{-1}$. This behavior in $k_{\mathrm{B}}$ could possibly be related to the observation that dilutions greater than $40 \times$ were needed to reduce particle-particle interactions in emulsions in order to accurately measure $r_{\text {Emul }}$ using DLS. However, the observed behavior for $k_{\mathrm{B}}$ shown in Fig. 4 a could also be due to the effects of "crowding" on the NMR-determined $k_{\mathrm{B}}$. To see this, consider a thought experiment where we label isoflurane molecules within a given emulsion droplet at $t=0$ and monitor their positions in time (these isofluranes are represented by red circles in Fig. 8). The average residence lifetime for an isoflurane molecule to stay within the same emulsion droplet that they were in at $t=0$ is given by $\tau_{\text {lifetime }}^{\text {Emul }}=\frac{1}{k_{\mathrm{B}}}$. After a time $\tau=\tau_{\text {lifetime, }}^{\text {Emul }}$, only $\mathrm{e}^{-1} \approx 36.8 \%$ of isoflurane molecules will still reside in the same emulsion droplet they were in at $t=0$. Those isoflurane molecules that left an emulsion droplet during the time $\tau=\tau_{\text {lifetime }}^{\text {Emul }}$ would be expected to diffuse from the surface of the emulsion droplet by an average diffusion length, $L_{\mathrm{D}}$, given by:

$$
L_{\mathrm{D}}=\sqrt{6 D_{\text {Iso,aq }} \tau_{\text {lifetime }}^{\text {Emul }}}=\sqrt{\frac{6 D_{\text {Iso,aq }}}{k_{\mathrm{B}}}}
$$

Isoflurane molecules that were released from an emulsion droplet will not enter a different emulsion droplet during the
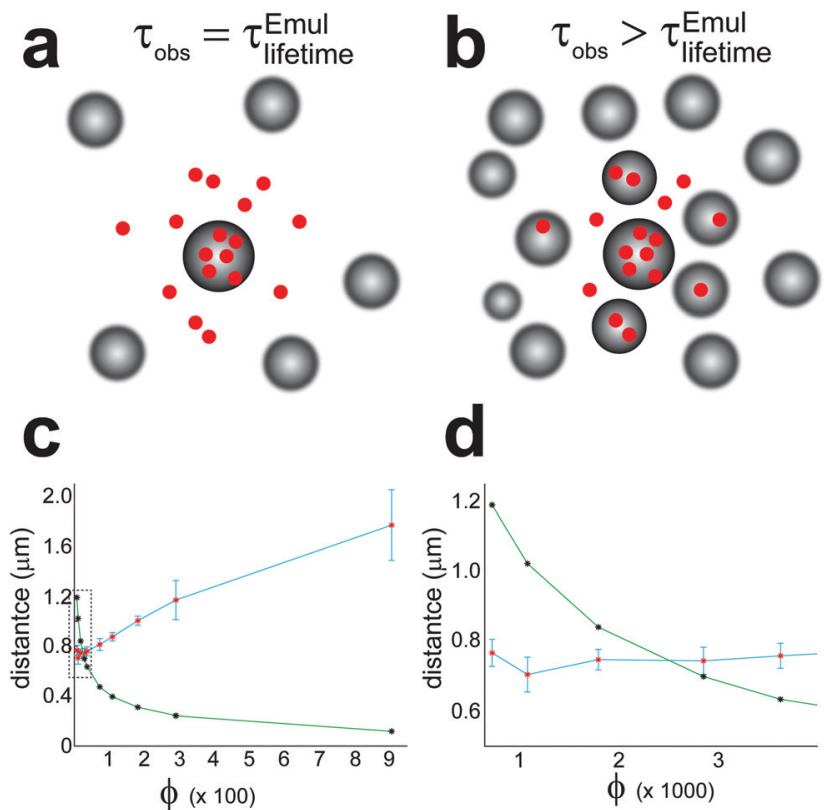

Fig. 8 The effects of crowding on the apparent reverse rate constant in eqn (18) determined from NMR, $k_{B}^{\text {Effective }}$. Isoflurane molecules within a central emulsion droplet at $t=0$ are represented by red circles. (a) Depiction of the "lifetime-limited regime" where $L_{\mathrm{D}}<R_{\mathrm{nn}}$. In this case, the residence lifetime of isoflurane molecules to be found in an emulsion droplet is the same as the residence lifetime of isoflurane molecules to remain in a given emulsion droplet, $\tau_{\text {obs }}=\tau_{\text {lifetime. }}^{\text {Emul }}$ (b) Depiction of the "crowded-regime" when $L_{\mathrm{D}}>R_{\mathrm{nn}}$. In this case, the observed residence lifetime of isoflurane to be in any emulsion droplet is greater than the actual residence lifetime to remain in a given droplet, i.e., $\tau_{\mathrm{obs}}>\tau_{\text {lifetime. }}^{\text {Emul }}$ (c) Plot of the experimental $R_{\mathrm{nn}}$ (green curve with black asterisks denoting $R_{\mathrm{nn}}$ calculated from eqn (26)) and $L_{\mathrm{D}}$ (blue curve with red asterisks denoting $L_{\mathrm{D}}$ calculated from eqn (25) using the $k_{\mathrm{B}}$ values in Fig. 4a) vs. the total organic phase volume fraction, $\phi$ in eqn (24). A dashed box is drawn around the crossover between "crowded" and "lifetime-limited regimes", which is shown more clearly in (d). The "lifetime-limited regime" occurs for $\phi \leq 0.0029$, in which case, the NMRdetermined $k_{\mathrm{B}}$ would correspond to the actual release rate constant of isoflurane from emulsion droplets.

time $\tau_{\text {lifetime }}^{\text {Emul }}$ as long as $L_{\mathrm{D}}$ is smaller than the shortest distance between neighboring emulsion droplets, $R_{\mathrm{nn}}$, which is given by:

$$
R_{\mathrm{nn}}=\left(\left(\frac{4 \pi}{3 \phi}\right)^{\frac{1}{3}}-2\right) r_{\text {Emul }}
$$

When $L_{\mathrm{D}}<R_{\mathrm{nn}}$, which we refer to as the "lifetime-limited" regime and is depicted in Fig. 8a, the residence lifetime of isoflurane molecules remaining in any emulsion droplet is just given by $\tau_{\text {obs }}=\tau_{\text {lifetime. }}^{\text {Emul }}$ In this case, the NMR-determined $k_{\mathrm{B}}$ value would correspond to the actual $k_{\mathrm{B}}$ value.

For $L_{\mathrm{D}}>R_{\mathrm{nn}}$, however, isoflurane molecules leaving an emulsion droplet would be able to diffuse and reenter neighboring droplets within a time $\tau_{\text {lifetime. }}^{\text {Emul }}$. This situation, which we refer to as the "crowded regime", is depicted in Fig. 8b. While the residence lifetime of an isoflurane molecule in a given emulsion droplet is still $\tau_{\text {lifetime }}^{\text {Emul }}$ as illustrated in Fig. 8, the apparent lifetime for an isoflurane molecule to remain in any emulsion droplet is longer as a result of isoflurane 
molecules diffusing into neighboring emulsion droplets, i.e., $\tau_{\text {obs }}=\frac{1}{k_{\mathrm{B}}^{\text {Effective }}}>\tau_{\text {lifetime }}^{\text {Emul }}=\frac{1}{k_{\mathrm{B}}}$. Since NMR is sensitive to the lifetime of isoflurane in different chemical environments overall, the observed isoflurane release rate constant determined by $\mathrm{NMR}, k_{\mathrm{B}}^{\mathrm{Effective}}$, would be smaller in the "crowded-regime" than the true isoflurane release rate constant, $k_{\mathrm{B}}^{\text {Effective }}<k_{\mathrm{B}}$.

In Fig. $8 \mathrm{c}$, the (blue curve) calculated $L_{\mathrm{D}}$ distances using the apparent $k_{\mathrm{B}}^{\text {Effective }}$ values given in Fig. 4 a as input in eqn (25) and the (green curve) calculated $R_{\mathrm{nn}}$ distances using eqn (26) are both plotted as a function of $\phi$. A crossover between the "crowded regime" $\left(L_{\mathrm{D}}>R_{\mathrm{nn}}\right)$ and the "lifetime-limited regime" $\left(L_{\mathrm{D}}<R_{\mathrm{nn}}\right)$ is observed around $\phi=0.0029$ (corresponding to a $50 \times$ dilution and denoted by the dashed box in Fig. 8c, which is shown more clearly in Fig. 8d). This crossover

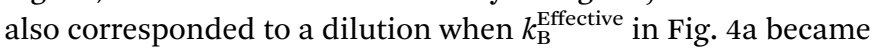
relatively constant, i.e., $k_{\mathrm{B}}^{\mathrm{Effective}} \approx k_{\mathrm{B}}$. While the above qualitative arguments could explain the observed [Emul]-dependence of $k_{\mathrm{B}}$ in Fig. 4a, calculations explicitly taking into account crowding effects and the diffusion of isoflurane between aqueous phase and multiple emulsion droplets ${ }^{36-38}$ could provide further support that the crossover between "crowded-" and "lifetime-limited" regimes affects the NMR determination of the actual kinetic parameters in emulsions.

\section{Conclusions}

The theory for determining the kinetic parameters describing molecules exchanging between emulsion droplets and an aqueous phase using the Bloch-McConnell equations was presented. Simple algebraic expressions were provided from which the kinetic parameters could be determined from the measured line widths and resonance frequencies of the exchanging molecule. As a demonstration, a series of dilution experiments were used to determine the kinetic exchange parameters of isoflurane in an emulsification of isoflurane and FC43. The isoflurane exchange was found to always be within the fast exchange regime, with a forward rate constant of isoflurane entering an emulsion droplet being on the same order of magnitude as the theoretical Smoluchowski diffusion-limited rate constant, indicating that isoflurane entering an emulsion droplet was diffusion-limited. The NMR-determined reverse rate "constant" for isoflurane release from emulsion droplets was shown to increase by a factor of three for dilution up to $50 \times$, after which $k_{\mathrm{B}}$ appeared relatively constant. It was argued that this behavior was a result of a crossover between "crowded-" and "lifetime-limited" regimes, whereby the NMR-determined $k_{\mathrm{B}}$ would correspond to the actual reverse rate constant only in the "lifetime-limited" regime. A small, downfield frequency shift of the isoflurane resonances with increasing [Iso $]_{\text {tot }}$ was also observed and provided experimental evidence in support of composition-dependence of the isoflurane chemical shifts used in the Bloch-McConnell simulations. The theory and methodology presented in this work should provide more insight into the interpretation of NMR spectra in exchanging systems where composition effects can be important, such as in nanoemulsions, hydrogels, etc. Compared to more traditional techniques, NMR exchange studies could provide alternative methods to more accurately characterize drug release rates in nanocarrier systems. ${ }^{27}$

\section{Conflicts of interest}

There are no conflicts to declare.

\section{Acknowledgements}

Research reported in this manuscript was supported by funding from the National Institute of Diabetes and Digestive and Kidney Diseases of the National Institutes of Health under award number R01DK116875, the Diabetes Research Institute Foundation, the National Science Foundation under CHE-1626015 and CHE-1807724, and the University of Miami Center for Computational Science. The content is solely the responsibility of the authors and does not necessarily represent the official views of the National Institutes of Health.

\section{Notes and references}

1 F. Goodarzi and S. Zendehboudi, Can. J. Chem. Eng., 2019, 97, 281-309.

2 D. A. Weitz and D. J. Pine, Dynamic Light Scattering: The Method and Some Applications, Clarendon Press, 1st edn, 1993.

3 P. S. Denkova, S. Tcholakova, N. D. Denkov, K. D. Danov, B. Campbell, C. Shawl and D. Kim, Langmuir, 2004, 20, 11402-11413.

4 P. Jokela, P. D. I. Fletcher, R. Aveyard and J.-R. Lu, J. Colloid Interface Sci., 1990, 134, 417-426.

5 B. P. Binks and M. Kirkland, Phys. Chem. Chem. Phys., 2002, 4, 3727-3733.

6 V. Klang, N. B. Matsko, C. Valenta and F. Hofer, Micron, 2012, 43, 85-103.

7 G. J. W. Goudappel, J. P. M. van Duynhoven and M. M. W. Mooren, J. Colloid Interface Sci., 2001, 239, 535-542.

8 M. L. Johns and K. G. Hollingsworth, Prog. Nucl. Magn. Reson. Spectrosc., 2007, 50, 51-70.

9 M. L. Johns, Curr. Opin. Colloid Interface Sci., 2009, 14, 178-183.

10 K. B. Sutradhar and M. L. Amin, Eur. J. Nanomed., 2013, 5, 97-110.

11 Y. Singh, J. G. Meher, K. Raval, F. A. Khan, M. Chaurasia, N. K. Jain and M. K. Chourasia, J. Controlled Release, 2017, 252, 28-49.

12 M. Levy and S. Benita, Int. J. Pharm., 1990, 66, 29-37.

13 L. Mora, K. Y. Chumbimuni-Torres, C. Clawson, L. Hernandez, L. Zhang and J. Wang, J. Controlled Release, 2009, 140, 69-73.

14 S. Petersen, A. Fahr and H. Bunjes, Mol. Pharmaceutics, 2010, 7, 350-363.

15 V. Agrahari, J. Meng, S. Purohit, N. Oyler and B.-B. Youan, J. Pharm. Sci., 2017, 106, 3005-3015.

16 K. I. Momot, P. W. Kuchel, B. E. Chapman, P. Deo and D. Whittaker, Langmuir, 2003, 19, 2088-2095. 
17 K. I. Momot and P. W. Kuchel, Concepts Magn. Reson., Part A, 2003, 19A, 51-64.

18 M. J. Hey and F. Al-Sagheer, Langmuir, 1994, 10, 1370-1376.

19 J. Wolber, I. J. Rowland, M. O. Leach and A. Bifone, Mag. Res. Med., 1999, 41, 442-449.

20 H. M. McConnell, J. Chem. Phys., 1958, 28, 430-431.

21 H. S. Gutowsky and A. Saika, J. Chem. Phys., 1953, 21, 1688-1694.

22 A. D. Bain, Prog. Nucl. Magn. Reson. Spectrosc., 2003, 43, 63-103.

23 A. G. Palmer and H. Koss, Methods Enzymol., 2019, 615, 177-236.

24 J. Jeener, B. H. Meier, P. Bachmann and R. R. Ernst, J. Chem. Phys., 1979, 71, 4546-4553.

25 D. Deng, H. Li, J. Yao and S. Han, Chem. Phys. Lett., 2003, 376, 125-129.

26 C. A. Fraker, A. J. Mendez, L. Inverardi, C. Ricordi and C. L. Stabler, Colloids Surf., B, 2012, 98, 26-35.
27 Z. Gong, M. H. Tootoonchi, C. A. Fraker and J. D. Walls, Int. J. Pharm., submitted.

28 S. J. Gibbs and C. S. Johnson, J. Mag. Res., 1991, 93, 395-402.

29 M. von Smoluchowski, Z. Phys. Chem., 1917, 92, 129-168.

30 L. Fielding, Prog. Nucl. Mag. Res., 2007, 51, 219-242.

31 D. Sinnaeve, Concepts Magn. Reson., Part A, 2012, 40, 39-65.

32 T. I. Igumenova, U. Brath, M. Akke and A. G. Palmer, J. Am. Chem. Soc., 2007, 129, 13396-13397.

33 P. Vallurpalli, D. F. Hansen and L. E. Kay, J. Am. Chem. Soc., 2008, 130, 2734-2735.

34 R. A. Reck and S. Prager, J. Chem. Phys., 1965, 42, 3027-3032.

35 B. U. Felderhof and J. M. Deutch, J. Chem. Phys., 1976, 64, 4551-4558.

36 P. S. Belton and B. P. Hills, Mol. Phys., 1987, 61, 999-1018. 37 P. S. Belton and B. P. Hills, Mol. Phys., 1988, 65, 313-326.

38 M. R. Gherase, J. C. Wallace, A. R. Cross and G. E. Santyr, J. Chem. Phys., 2006, 125, 044906. 